\title{
High-Throughput Acoustofluidic Fabrication of Tumor Spheroids
}

Bin Chen, $1,2,6$ Yue Wu,1,6 Zheng Ao,1 Hongwei Cai, 1 Asael Nunez,1 Yunhua Liu,3,5 John Foley,4,5 Kenneth Nephew,4,5 Xiongbin Lu,3,5 and Feng Gu0 1,5

1. Department of Intelligent Systems Engineering, Indiana University, Bloomington, IN 47405, United States

2. Department of Laboratory Medicine, Guangzhou First People's Hospital, School of Medicine, South China University of Technology, Guangzhou 510180, China.

3. Department of Medical and Molecular Genetics, Indiana University School of Medicine, Indianapolis, IN 46202, United States

4. Cell, Molecular and Cancer Biology Program, Medical Sciences, Indiana University School of Medicine, Bloomington, IN 47405, United States

5. Melvin and Bren Simon Cancer Center, Indiana University School of Medicine, Indianapolis, IN 46202, United States

6. These authors contributed equally to this work

*Corresponding email: fengguo@iu.edu

Abstract. Three-dimensional (3D) culture of multicellular spheroids, offering a desirable biomimetic microenvironment, is appropriate for recapitulating tissue cellular adhesive complexity and revealing a more realistic drug response. However, current 3D culture methods are suffering from low-throughput, poor controllability, intensive-labor, and variation in spheroid size, thus not ready for many high-throughput screening applications including drug discovery and toxicity testing. Herein, we developed a high-throughput multicellular spheroid fabrication method using acoustofluidics. By acoustically-assembling cancer cells with low-cost and disposable devices, our method can produce more than 12,000 multicellular aggregates within several minutes and allow us to transfer these aggregates into ultra-low attachment dishes for long-term culture. This method can generate more than 6,000 tumor spheroids per operation, and reduce tumor spheroid formation time to one day. Our platform has advantages in forming spheroids with high throughput, short time, and long-term effectiveness, and is easy-to-operation. This acoustofluidic spheroid assembly method provides a simple and efficient way to produce large numbers of uniform-sized spheroids for biomedical applications in translational medicine, pharmaceutical industry and basic life science research.

Key Words: 3D Cell Culture; Tumor Spheroids; Acoustic Assembly; Acoustofluidics;

This is the author's manuscript of the article published in final edited form as:

Chen, B., Wu, Y., Ao, Z., Cai, H., Nunez, A., Liu, Y., Foley, J., Nephew, K., Lu, X., \& Guo, F. (2019). High-throughput acoustofluidic fabrication of tumor spheroids. Lab on a Chip, 19(10), 1755-1763. https://doi.org/10.1039/C9LC00135B 


\section{Introduction}

Traditional in vitro drug screening, normally based on 2D cell cultures on plastic surfaces, 1,2 cannot recapitulate the in vivo physiological conditions, such as the complex microenvironment due to the lack of appropriate cell-cell and cell-matrix interactions and absence of tissue-specific architecture, mechanical, and chemical cues that are essential for unique functions of tissues and organs in the body.3 The lack of physical-biochemical characteristics of the cell monolayer generally leads to greater efficiency of drugs reaching specific molecular targets, which in turn contributes to the high failure rate of compounds at the latter stages of dug development. To overcome this limitation, 3D cell culture methods have been developed to better represent the in vivo microenvironment and mimic the physiological functions of living tissues when investigating the response to therapeutic agents.4, 5

3D tumor spheroid culture is considered as an improved in vitro model to mimic biological properties of poorly vascularized regions of tumors and non-vascularized micro-metastases since they retain the architecture and many morphological and physiological characteristics of their tumor counterparts. 6-8 Spheroid formation is one of the most well characterized models for 3D culture and screening due to its simplicity, reproducibility, and similarity to physiological tissues as compared to other scaffold-based methods.9, 10 Spheroids are self-assembled spherical clusters of cell colonies cultured in environments where cell-cell interactions dominate over cellsubstrate interactions, and they naturally mimic avascular tumors with inherent metabolic (oxygen) and proliferative (nutrient) gradients.11-16

Although tumor spheroids have been widely recognized and served as excellent physiologic tumor models17, it has been difficult to scale up spheroid culture for screening and testing. Traditional spheroid formation and culture systems used hanging drops18, non-adherent surfaces19, spinner flask cultures20, scaffold structures21, 22 or micro/nanostructures23. However, these methods require complex set-up, are time-consuming, low-throughput, and low-yielded, and produce spheres of irregular size leading to many difficulties in scale up. Recently, various microfluidic devices have been developed to increase spheroid formation efficiency and simplify handling procedures.24-31 The significant advantages of microfluidic devices include providing controlled mixing, chemical concentration gradients, lower reagent consumption, continuous perfusion and precision control of shear stress on cells.32-39 Many of these techniques, however, still suffer from problems such as inability to support long-term culture and incompatibility with high-throughput drug screening. 
Acoustics provides a promising alternative approach for spheroid fabrication, benefiting from its excellent biocompatibility, flexibility, and contactless and label-free manipulation of cells while preserving the cells' native state.40-51 In this paper, we describe a high-throughput acoustic spheroid fabrication method in a simple disposable polydimethylsiloxane (PDMS) device. In comparison with conventional 3D spheroid formation methods, the developed platform has the following advantages: (i) high generation yield and reproducibility, (ii) fast spheroid assembly with controllable size, (iii) long term culture that maintained high viability, (iv) applicability to a wide range of cells lines, and (v) high-throughput drug screening with close-to-physiological medium flow conditions.

\section{Results and discussion}

Working mechanism: We developed a high-throughput cell spheroid fabrication method as a new advance of our early work on surface acoustic waves (SAW) and acoustofluidics.52-55 The spheroid fabrication device can be assembled by coupling a disposable PDMS device with multiple cell assembly channels onto a reusable standing surface acoustic wave (SSAW) generator using a thin layer of oil. The PDMS devices were fabricated by bonding a thick PDMS stamp with parallel spheroid assembly channels onto a thin PDMS layer (Fig. 1(a)). Once the radio frequency (RF) signal was applied, a SSAW field was generated by the SSAW generator, propagated on the piezoelectric substrate, and leaked into the PDMS channels through the thin layer of coupling oil between the SSAW generator and a PDMS layer. When the SSAW entered into the PDMS channels and met with the liquid, a linear pressure node distribution was formed inside of each channel. The suspended single cells were moved towards and trapped into the sounding pressure node under the driven of acoustic radiation forces in each channel (Fig.1(b)). With a 3 min acoustofluidic treatment, randomly distributed cells were moved to the nearby pressure nodes and acoustically-assembled into 3D multicellular aggregates in a large-scale dotarray pattern. In our experiments, we generated 12,000 pressure nodes at the same time within one PDMS device, resulting in 12,000 cell aggregates. After an on-chip incubation, these 3D cell aggregates were developing cell-cell contacts and self-assembled into mature aggregates. These cell aggregates could be washed from the disposable PDMS device and cultured in the ultra-low attachment dishes, while the SSAW generator could be reused for another spheroid assembly experiment.

High-throughput and short time spheroid formation: In our previous work, we demonstrated the generation of cell spheroids using directly bonded acoustofluidic devices or disposable glass 
capillaries coupled with standing acoustic waves, however, the throughput of cell spheroid generation was limited at around $300.56,57$ In this work, we developed a new strategy to significantly increase the throughput of cell spheroid production from 300 to 6,000 . In our experiments, we generated surface acoustic waves with a wavelength of $300 \mu \mathrm{m}$. The PDMS device was designed with 60 parallel channels and each channel with a dimension of $3 \mathrm{~cm} \times 150$ $\mu \mathrm{m} \times 150 \mu \mathrm{m}$. Thus, about 12,000 pressure nodes could be formed at the same time within a PDMS device. The suspended cells in each channel can be moved towards and captured in the sounding pressure node as a 3D trap with a dimension of $150 \mu \mathrm{m} \times 150 \mu \mathrm{m} \times 150 \mu \mathrm{m}$ resulting in 12,000 cell aggregates on the whole device (Fig. 1 (c)-(d)). In our experiments, mouse pancreatic cancer cell line-Panc0258 were injected in the PDMS device. Before turning on the acoustic field, all the cells were randomly distributed in each microfluidic channel. After applying the acoustic field, the Panc02 cells were trapped into 3D cell aggregates in a large-scale, dot-array format (Fig. 2(a)-(b)).

To maintain the cell aggregates during transfer from the PDMS device into the ultra-low attachment petri-dish, we optimized the on-chip incubation time from 1 to 5 hours. If the incubation time was more than 2 hours for cell-cell adhesion, Panc02 spheroids remained largely intact after this transfer process, at an efficiency of approximately $50 \%(50.54 \pm 1.43 \%$ to $52.75 \pm 2.58 \%)$ (Figure 2(c)). Thus, we determined 2 hours as the optimal incubation time for cells aggregates to form cell-cell contacts. With our acoustofluidic design, 12,000 cell clusters could be generated per operation. The transfer of the acoustic assembly of cell clusters from the device to an ultralow attachment dish yielded more than 6,000 cell spheroids after one day culture (Figure 2(d)) and provided a much better production throughput with shorter formation time than other methods (Table 1).

Additionally, we also compared our method with commercially available methods such as Corning ${ }^{\circledR}$ spheroid microplates. Tumor spheroid cultures were visualized at day 0 , day 0.5 , day 1, day 3 , day 5, and day 7. In Fig.3, Panc02 cells started to form spheroids at 12 hours using our acoustic assembly approach, whereas spheroids were not formed until day 3 using commercial microplates (Fig. 3). This suggests that our acoustic assembly platform has the ability to accelerate spheroid formation, likely due to the initial compact cell aggregate formation by acoustics. Therefore, we demonstrated a tumor spheroid formation technique with a greater highthroughput and efficiency.

Tumor spheroid characterization: To characterize the developed cell spheroids, mouse pancreatic cancer cell line-Panc02 cells were acoustically-assembled into cell spheroids as 
described above and cultured for 7 days. Cell viability and proliferation rates were characterized using live/dead staining and cell counting kit-8 tests. Fig.4(a) and Fig.4(b) showed the live/dead staining of Panc02 cell spheroids over a 7-day culture, indicating that the majority of cells (>90\%) remained viable after 7 days of culture. Cell proliferation was assessed using the cell counting kit8. The cell number inside the Panc02 spheroids increased dramatically during the 7-day culture indicating a healthy proliferation in the dish (Fig. 4(c)). In addition, the size uniformity of the Panc02 cell spheroids was characterized from Day 1 to Day 3. Fig.4(d) showed the size distribution of cell spheroids. As seen, the cell spheroids formed by our method had good uniformity initially, and size difference increases as spheroids start to merge occasionally during the boundary and matrix free culture (Fig. 2(d)).

To apply our method for various cell lines, we chose six different types of mouse and human cancer cell lines. As shown in Fig.5, murine pancreatic cancer cell lines Panc02, UN-KC-6141, murine breast cancer cell line EO771 and human breast cancer cell line MCF-7cell spheroids showed smooth boundary and compact spheroidal shape after 7 days of culture, whereas human ovarian carcinoma cell line A2780, and human lung cancer cell line A549 formed only loose aggregates after 7 days of culture. The aggregates of human cancer cell lines can be easily disrupted mechanically by pipetting, suggesting the cell-cell contacts established by these cultures are weak. This difference between mouse and human cancer cell line spheroids is consistent with other reports using scaffold free spheroid formation methods. 8 This observed spheroid morphology did not change in cultures maintained up to 14 days. This suggests that our acoustic cell assembly system produces both for both human and murine spheroids with characteristics consistent with lower throughput methodologies.

In vitro spheroids have been reported to reflect in vivo nutrient, cytokine and oxygen distribution. It has been reported that large spheroids consist of proliferative, quiescent and necrotic zones.11 Cells in the necrotic core have disintegrated nuclei and membranes, and they lose their activities due to nutrients starvation and toxic waste accumulation. Beyond the diffusive limit, metabolic wastes are accumulated in the inner layer of the spheroids, leading to the formation a necrotic core. To examine the existence of hypoxia, the tumor spheroids were stained with the Image-IT TM Hypoxia Reagent. As shown in Fig.6, spheroids of mouse embryonic cell line P19, and mouse pancreatic cancer cell lines Panc02 and UN-KC-6141 were cultured for 10 days to allow for formation of the hypoxic core. As indicated by the staining, hypoxic cores were observed in the center of the spheroid. The hypoxic centers of the spheroids were similar to those reported for other assembly methods 12 . 
Drug testing: We further demonstrated the compound screening with assembled tumor spheroids using our acoustofluidic method. As Gemcitabine is the most common chemotherapy used to pancreatic cancer,59 we evaluated its efficacy against Panc02 spheroids. Gemcitabine was applied in a range of $0.1 \mu \mathrm{M} \sim 100 \mu \mathrm{M}$. Parallel experiments were set with traditional 2D monolayer cell cultures to examine differential response of 2D and 3D cell cultures. Cell number and viability were measured using MTT assay after 2 days of Gemcitabine treatment at the concentrations of $0.1,1,10,25,50$, and $100 \mu \mathrm{M}$. The IC50 value was calculated. For the monolayer culture, the IC50 of Gemcitabine on Panc02 cells was $9.99 \pm 0.07 \mu \mathrm{M}$ while the IC50 value for spheroids was $18.38 \pm 0.09 \mu \mathrm{M}$ (Figure 7). From the regression curves, both spheroids and monolayer cultures showed a dose-dependent response to the drugs. This indicates that Panc02 cells grown in the form of spheroids retained dosage-dependent cytotoxicity of the drug. However, spheroids were shown to confer a higher resistance to Gemcitabine treatment in comparison to its monolayer counterpart, which could be attributed to several characteristics of 3D culture as investigated in other studies.60

\section{Experimental}

Cell preparation: Human breast cancer cell line MCF-7, human lung cancer cell line A549, human ovarian cancer cell line A2780, and murine embryonic carcinoma cell line P19 were purchased from the American Type Culture Collection (ATCC, Rockville, MD). MCF-7 cells, A549 cells, Panc02 cells, and UN-KC-6141 cells were maintained in Dulbecco's modified Eagle medium (GIBCO), supplemented with $10 \%$ fetal bovine serum (FBS; GIBCO) and $100 \mathrm{U} / \mathrm{mL}$ penicillin/streptomycin (P/S; GIBCO). A2780 cells were maintained in RPMI-1640 medium (GIBCO), supplemented with $10 \%$ fetal bovine serum (FBS; GIBCO) and $100 \mathrm{U} / \mathrm{mL}$ penicillin/streptomycin (P/S; GIBCO). P19 cells were cultured in Alpha Modified Eagle's Medium (Corning, NY) supplemented with $10 \%$ fetal bovine serum (FBS; Gibco) and 100U/mL penicillin/streptomycin (P/S; Gibco). All cells were cultured in a humidified incubator supplemented with $5 \% \mathrm{CO}_{2}$ at $37^{\circ} \mathrm{C}$. Cell suspensions were made by dissociating cells with $0.25 \%$ trypsin-EDTA (Gibco 25200, Invitrogen Co.), centrifuging dissociated cells at $400 \mathrm{~g}$ for $5 \mathrm{~min}$ at room temperature and re-suspending in culture media. Cell density was enumerated using a hemocytometer.

Device fabrication: Microfluidic device was produced by soft lithography from Sylgard 184 polydimethylsiloxane (PDMS) using molds made with SU-8.61, 62 After drilling holes for an inlet and outlet with a puncher ( $1 \mathrm{~mm}$ diameter, Harris Uni-Core, USA), we took advantage of oxygen 
plasma treatment (PDC001, Harrick Plasma, USA) to bind the molded PDMS chamber to a thin layer of PDMS film (25 $\mu \mathrm{m}$ thickness) that was spin coated on a surface polished silicon wafer.

The SSAW generator was fabricated by a standard soft lithography and lift-off process. A 7- $\mu \mathrm{m}-$ thick photoresist layer (S1813, MicroChem, USA) was spin-coated on the piezoelectric substrate (a 500- $\mu \mathrm{m}$-thick, double-side polished, $128^{\circ} \mathrm{YX}$-propagation $\mathrm{LiNbO}_{3}$ wafer). Then, the designed IDT patterns of 40 electrode pairs with the $75 \mu \mathrm{m}$ finger width and periodic spacing were transferred from the plastic mask (Kunshan Kaisheng Electronics Co., Ltd, China) to the substrate by UV exposure. The IDT patterns were developed in a photoresist developer (MF CD-26, Microposit, USA) and deposited with double metal layers ( $\mathrm{Cr} / 50 \AA$, Au/600 $\AA$ ) by a thermal evaporation (JSD-350, Anhui Jiaoshuo Vacuum Technology Co., Ltd, China). IDTs on the piezoelectric substrate were finally obtained after a standard lift-off process. Then the resonant frequency of the fabricated SSAW generator was measured at around 13.13-13.41 MHz using a network analyzer (E8362C, Agilent, USA).

Prior to the cell assembly, the PDMS device was sterilized by autoclaving at $121^{\circ} \mathrm{C}$ for $30 \mathrm{~min}$. During experiment manipulation, the disposable PDMS device was assembled on top of a SSAW substrate with a layer of coupling oil. The mineral oil (SLBX1961, SIGMA, USA) was chosen as the coupling material to introduce the acoustic waves from the substrate to the above device. In comparison with water or olive oil or other coupling materials, mineral oil can offer both a lower evaporation rate and a better coupling performance with causing no harm to cell viability.

High-throughput acoustic cell assembly: In the cell patterning experiment, cells were aggregated into linear assembly arrays in PDMS channels by applying a radio frequency signal produced by a function generator (AFG3102C, Tektronix, USA) and modulated with an amplifier (25A100A, Amplifier Research, USA) to the IDT pair. The movement of cells was monitored and recorded by a microscope (IX-81, Olympus, Japan) with a CMOS camera (ORCA-Flash 4.0, HAMAMATSU, Japan) connected to a computer (Cellsens) The input voltages on the devices were from 10 to $60 \mathrm{Vpp}$. Different kinds of cells were resuspended in type I collagen (Life Technologies, USA) and injected after the acoustic field was formed. The whole acoustic cell aggregation process took one minute. To form cell spheroids, the cell clusters were incubated in PDMS device for a short period and subsequently transferred into ultra-low attachment plates (Corning, 3471, USA) with fresh cell culture medium. The cell cluster plate was incubated and maintained at $5 \% \mathrm{CO}_{2}$ and $37{ }^{\circ} \mathrm{C}$. These cell clusters were imaged and recorded every day for several days until spheroids like structure formed. 
Spheroid formation by Corning ${ }^{\circledR}$ spheroid microplates: Panc02 cells used in this study were harvested as single cell suspension with $0.25 \%$ Trypsin-EDTA solution (Gibco 25200, Invitrogen Co) for $2 \mathrm{~min}$ at $37^{\circ} \mathrm{C}$, centrifuged at $400 \mathrm{~g}$ for $5 \mathrm{~min}$ and resuspended in their respective culture media. To induce 3D spheroid formation, the harvested cells were seeded at fixed density of 500 cells per well into Corning® spheroid microplates (Corning, USA), spun down at $400 \mathrm{~g}$ for $3 \mathrm{~min}$ and incubated at $37{ }^{\circ} \mathrm{C}, 5 \% \mathrm{CO}$. Spheroid cultures were visualized at $0 \sim 7$ days using a microscope.

Tumor spheroid proliferation and viability tests: Cell proliferation was assessed using the cell counting kit-8 (CCK-8; Sigma-Aldrich, St. Louis, MO, USA) according to the manufacturer's instructions. Control cells were exposed to an equivalent amount of vehicle. Tumor spheroids or cultured tumor cells were incubated in the CCK-8 solution for $4 \mathrm{~h}$, and the supernatants were transferred to 96 -well plates. Cell proliferation was assessed by measuring the absorbance at 450 $\mathrm{nm}$ using the Epoch ${ }^{\mathrm{TM}}$ microplate spectrophotometer (Bio-Tek Instruments, USA). The viability testing was achieved by the live/dead stain assay (live/dead viability kit, L3224, Thermo Fisher Scientific Inc.). Tumor spheroids were stained with a mixture of $2 \mu \mathrm{M}$ Calcein $\mathrm{AM}$ and $4 \mu \mathrm{M}$ ethidium homodimer-1 to stain for live and dead cells, respectively. Brightfield and fluorescent images were captured using a microscope (IX83, Olympus, Japan).

Tumor spheroids hypoxia characterization: To examine hypoxia, the tumor spheroids were stained with the Image-IT ${ }^{\mathrm{TM}}$ Hypoxia Reagent (Invitrogen ${ }^{\mathrm{TM}}$, USA) according to the manufacturer's instruction. The reagent was added to the spheroids at a final concentration of 10 $\mu \mathrm{M}$ and incubate at $37^{\circ} \mathrm{C}$ for 48 hours. The spheroids were then imaged on the fluorescence microscope with excitation/emission of $490 / 610 \mathrm{~nm}$.

Cytotoxicity assay: The cytotoxic effect of the chemotherapeutic drug Gemcitabine (LC Laboratories, Woburn, MA, USA) on Panc02 cell spheroids culture was compared to the cytotoxic effect on monolayer culture. For the cytotoxic assay, $1 \times 10_{4}$ cells suspended in complete medium were seeded in each well of a 96-well plate. The 4-day-old Panc02 spheroids were transferred to a new 96 ultra-low attachment well plate. The next day, the cells and spheroids were treated with different concentrations of Gemcitabine solution $(0,0.1,1,10,25,50,100 \mu \mathrm{M})$ in sextuplicate wells. After incubating the cells with Gemcitabine for $48 \mathrm{~h}, 20 \mu \mathrm{l}$ of MTT solution $(5 \mathrm{mg} / \mathrm{ml})$ was added into each well, and the cells were incubated for 4 hours. While the monolayer culture was left untouched in the original plate, the content of each well containing the tumor spheroids culture was transferred to a new, flat bottom 96-well plate before the plate was centrifuged at $200 \times \mathrm{g}$ for 5 minutes. Then, $100 \mu$ of media was aspirated from each well from the plates containing the 
monolayer and spheroids cultures. The plates were then blotted dry on paper towels, followed by the addition of $100 \mu \mathrm{l}$ of DMSO. Finally, absorbance was recorded at $570 \mathrm{~nm}$ using the Epoch ${ }^{\mathrm{TM}}$ microplate spectrophotometer (Bio-Tek Instruments, USA). The assay was carried out with 3 replicates for each culture.

Statistical analysis: Data presented in this study are representative of at least three independent experiments. All values are expressed as arithmetic mean \pm standard deviation (SD). Statistical difference between experimental groups was determined using Student's t test, when $P$ values $<0.05$ were considered statistically significant.

\section{Conclusions}

Our study presents a proof-of-concept in improving the cell spheroid formation throughout and time using acoustofluidics, for the purpose of drug efficacy, metabolism and toxicity studies. Compared to the traditional spheroid formation and culture methods, our approach generates a larger number of spheroids in a short time. Like other spheroid methods, the scaffold-free nature of the presented approach has the potential to eliminate the effects associated with stiffness, roughness and chemical composition of these substrates that affect cell behavior and phenotype. We expect that the acoustofluidic cell assembly technique can be used as a powerful tool for highthroughput screening in new drug development processes.

\section{Conflicts of interest}

The authors declare no conflicts of interests.

\section{Acknowledgments}

This project was supported by the departmental start-up fund and the Vice Provost for the Research through the Faculty Research Support Program (Indiana University Bloomington). Murine pancreatic cancer cell line Panc02 was a kind grift from Dr. Jill Smith (Georgetown University), and UN-KC-6141 cell line was a kind gift from Surinder K. Batra (University of Nebraska) 


\section{References}

1. W. Mueller-Klieser, American Journal of Physiology-Cell Physiology, 1997, 273, C1109-C1123.

2. A. Abbott, Nature, 2003, 424, 870.

3. F. Pampaloni, E. G. Reynaud and E. H. K. Stelzer, Nat Rev Mol Cell Bio, 2007, 8, 839-845.

4. L. G. Griffith and M. A. Swartz, Nat Rev Mol Cell Bio, 2006, 7, 211.

5. C. S. Shin, B. Kwak, B. Han and K. Park, Molecular Pharmaceutics, 2013, 10, 2167-2175.

6. $\quad$ R. Z. Lin and H. Y. Chang, Biotechnol J, 2008, 3, 1172-1184.

7. J. Friedrich, C. Seidel, R. Ebner and L. A. Kunz-Schughart, Nature Protocols, 2009, 4, 309.

8. B. W. Huang and J. Q. Gao, J Control Release, 2018, 270, 246-259.

9. L. P. Ferreira, V. M. Gaspar and J. F. Mano, Acta Biomater, 2018, 75, 11-34.

10. Y. B. Lee, E. M. Kim, H. Byun, H. K. Chang, K. Jeong, Z. M. Aman, Y. S. Choi, J. Park and H. Shin, Biomaterials, 2018, 165, 105-120.

11. E. Fennema, N. Rivron, J. Rouwkema, C. van Blitterswijk and J. de Boer, Trends Biotechnol, 2013, 31, 108-115.

12. M. W. Laschke and M. D. Menger, Biotechnol Adv, 2017, 35, 782-791.

13. K. M. Tevis, Y. L. Colson and M. W. Grinstaff, Adv Biosyst, 2017, 1.

14. D. L. Priwitaningrum, J. G. Blonde, A. Sridhar, J. van Baarlen, W. E. Hennink, G. Storm, S. Le Gac and J. Prakash, J Control Release, 2016, 244, 257-268.

15. M. Vinci, S. Gowan, F. Boxall, L. Patterson, M. Zimmermann, W. Court, C. Lomas, M. Mendiola, D. Hardisson and S. A. Eccles, BMC Biology, 2012, 10, 29.

16. J. Friedrich, R. Ebner and L. A. Kunz-Schughart, International Journal of Radiation Biology, 2007, 83, 849-871.

17. S. Nath and G. R. Devi, Pharmacology \& Therapeutics, 2016, 163, 94-108.

18. Y. C. Tung, A. Y. Hsiao, S. G. Allen, Y. S. Torisawa, M. Ho and S. Takayama, Analyst, 2011, 136, 473-478.

19. A. P. Napolitano, D. M. Dean, A. J. Man, J. Youssef, D. N. Ho, A. P. Rago, M. P. Lech and J. R. Morgan, Biotechniques, 2007, 43, 494, 496-500.

20. M. Ingram, G. B. Techy, R. Saroufeem, O. Yazan, K. S. Narayan, T. J. Goodwin and G. F. Spaulding, In Vitro Cellular \& Developmental Biology - Animal, 1997, 33, 459-466.

21. S. Yan, J. Wei, Y. Liu, H. Zhang, J. Chen and X. Li, Acta Biomater, 2015, 28, 138-148.

22. R. H. Dosh, A. Essa, N. Jordan-Mahy, C. Sammon and C. L. Le Maitre, Acta Biomaterialia, 2017, 62, 128-143.

23. Y. Yoshii, T. Furukawa, A. Waki, H. Okuyama, M. Inoue, M. Itoh, M. R. Zhang, H. Wakizaka, C. Sogawa, Y. Kiyono, H. Yoshii, Y. Fujibayashi and T. Saga, Biomaterials, 2015, 51, 278-289.

24. C. T. Kuo, J. Y. Wang, Y. F. Lin, A. M. Wo, B. P. C. Chen and H. Lee, Sci Rep, 2017, 7, 4363.

25. V. Vickerman, J. Blundo, S. Chung and R. Kamm, Lab Chip, 2008, 8, 1468-1477.

26. A. P. Aijian and R. L. Garrell, J Lab Autom, 2015, 20, 283-295.

27. Y. S. Torisawa, B. H. Chueh, D. Huh, P. Ramamurthy, T. M. Roth, K. F. Barald and S. Takayama, Lab Chip, 2007, 7, 770-776.

28. C. T. Kuo, S. R. Lu, W. M. Chen, J. Y. Wang, S. C. Lee, H. H. Chang, A. M. Wo, B. P. C. Chen and H. Lee, Lab Chip, 2018, 18, 2453-2465.

29. J. Park, G. H. Lee, J. Yull Park, J. C. Lee and H. C. Kim, Biofabrication, 2017, 9, 045006.

30. L. Y. Yeo, H. C. Chang, P. P. Chan and J. R. Friend, Small, 2011, 7, 12-48.

31. A. Neild, S. Oberti and J. Dual, Sensors and Actuators B: Chemical, 2007, 121, 452-461.

32. S. Hao, L. Ha, G. Cheng, Y. Wan, Y. Xia, D. M. Sosnoski, A. M. Mastro and S. Y. Zheng, Small, 2018, 14, e1702787. 
33. C. M. Puleo, H. C. Yeh and T. H. Wang, Tissue Eng, 2007, 13, 2839-2854.

34. D. D. Nalayanda, C. M. Puleo, W. B. Fulton, T. H. Wang and F. Abdullah, Exp Lung Res, 2007, 33, 321-335.

35. J. Friend and L. Y. Yeo, Reviews of Modern Physics, 2011, 83, 647-704.

36. L. Trung-Dung and N. Nam-Trung, Micro and Nanosystems, 2010, 2, 217-225.

37. L. Meng, F. Cai, Q. Jin, L. Niu, C. Jiang, Z. Wang, J. Wu and H. Zheng, Sensors and Actuators B: Chemical, 2011, 160, 1599-1605.

38. H. D. Xi, H. Zheng, W. Guo, A. M. Ganan-Calvo, Y. Ai, C. W. Tsao, J. Zhou, W. Li, Y. Huang, N. T. Nguyen and S. H. Tan, Lab Chip, 2017, 17, 751-771.

39. L. Y. Yeo and J. R. Friend, Biomicrofluidics, 2009, 3, 12002.

40. D. J. Collins, B. Morahan, J. Garcia-Bustos, C. Doerig, M. Plebanski and A. Neild, Nat Commun, 2015, 6, 8686.

41. F. Cai, Z. He, Z. Liu, L. Meng, X. Cheng and H. Zheng, Applied Physics Letters, 2011, 99, 253505.

42. D. J. Collins, A. Neild and Y. Ai, Lab Chip, 2016, 16, 471-479.

43. L. Y. Yeo and J. R. Friend, Annual Review of Fluid Mechanics, 2014, 46, 379-406.

44. K. H. Lam, H. S. Hsu, Y. Li, C. Lee, A. Lin, Q. Zhou, E. S. Kim and K. K. Shung, Biotechnol Bioeng, 2013, 110, 881-886.

45. T.-D. Luong, V.-N. Phan and N.-T. Nguyen, Microfluidics and Nanofluidics, 2010, 10, 619-625.

46. L. Meng, F. Cai, J. Chen, L. Niu, Y. Li, J. Wu and H. Zheng, Applied Physics Letters, 2012, 100, 173701.

47. W. Qi, R. Li, T. Ma, J. Li, K. Kirk Shung, Q. Zhou and Z. Chen, Appl Phys Lett, 2013, 103, 103704.

48. Q. Zhou, S. Lau, D. Wu and K. K. Shung, Prog Mater Sci, 2011, 56, 139-174.

49. L. Meng, F. Cai, Z. Zhang, L. Niu, Q. Jin, F. Yan, J. Wu, Z. Wang and H. Zheng, Biomicrofluidics, 2011, 5, 044104.

50. Z. Ma, D. J. Collins and Y. Ai, Analytical Chemistry, 2016, 88, 5316-5323.

51. J. Reboud, R. Wilson, Y. Zhang, M. H. Ismail, Y. Bourquin and J. M. Cooper, Lab on a Chip, 2012, 12, 1268-1273.

52. F. Guo, P. Li, J. B. French, Z. Mao, H. Zhao, S. Li, N. Nama, J. R. Fick, S. J. Benkovic and T. J. Huang, Proc Natl Acad Sci U S A, 2015, 112, 43-48.

53. F. Guo, Z. M. Mao, Y. C. Chen, Z. W. Xie, J. P. Lata, P. Li, L. Q. Ren, J. Y. Liu, J. Yang, M. Dao, S. Suresh and T. J. Huang, $P$ Natl Acad Sci USA, 2016, 113, 1522-1527.

54. X. Ding, P. Li, S.-C. S. Lin, Z. S. Stratton, N. Nama, F. Guo, D. Slotcavage, X. Mao, J. Shi, F. Costanzo and T. J. Huang, Lab on a Chip, 2013, 13, 3626-3649.

55. F. Guo, Y. Xie, S. Li, J. Lata, L. Ren, Z. Mao, B. Ren, M. Wu, A. Ozcelik and T. J. Huang, Lab on a Chip, 2015, 15, 4517-4523.

56. K. Chen, M. Wu, F. Guo, P. Li, C. Y. Chan, Z. Mao, S. Li, L. Ren, R. Zhang and T. J. Huang, Lab Chip, 2016, 16, 2636-2643.

57. W. Yue, A. Zheng, C. Bin, M. Maram, B. Maria, L. Xiongbin and G. Feng, Nanotechnology, 2018, 29, 504006.

58. J. P. Smith, S. Wang, S. Nadella, S. A. Jablonski and L. M. Weiner, Cancer Immunology, Immunotherapy, 2018, 67, 195-207.

59. C. J. Campen, T. Dragovich and A. F. Baker, American Journal of Health-System Pharmacy, 2011, 68, 573.

60. M. P. Torres, S. Rachagani, J. J. Souchek, K. Mallya, S. L. Johansson and S. K. Batra, PLoS One, 2013, 8, e80580.

61. D. C. Duffy, J. C. McDonald, O. J. A. Schueller and G. M. Whitesides, Analytical Chemistry, 1998, 70, 4974-4984. 
62. P. Abgrall, V. Conedera, H. Camon, A. M. Gue and N. T. Nguyen, Electrophoresis, 2007, 28, 45394551.

63. D. A. Close, D. P. Camarco, F. Shan, S. J. Kochanek and P. A. Johnston, in High Content Screening: A Powerful Approach to Systems Cell Biology and Phenotypic Drug Discovery, eds. P. A. Johnston and O. J. Trask, Springer New York, New York, NY, 2018, DOI: 10.1007/978-1-4939-7357-6_20, pp. 355-369.

64. J. H. Lee and W. Hur, Biotechnology and Bioengineering, 2014, 111, 1038-1047.

65. T.-J. Lee, S. H. Bhang, W.-G. La, H. S. Yang, J. Y. Seong, H. Lee, G.-I. Im, S.-H. Lee and B.-S. Kim, Biotechnology Letters, 2011, 33, 829-836. 


\section{Figures and captions}

(a)

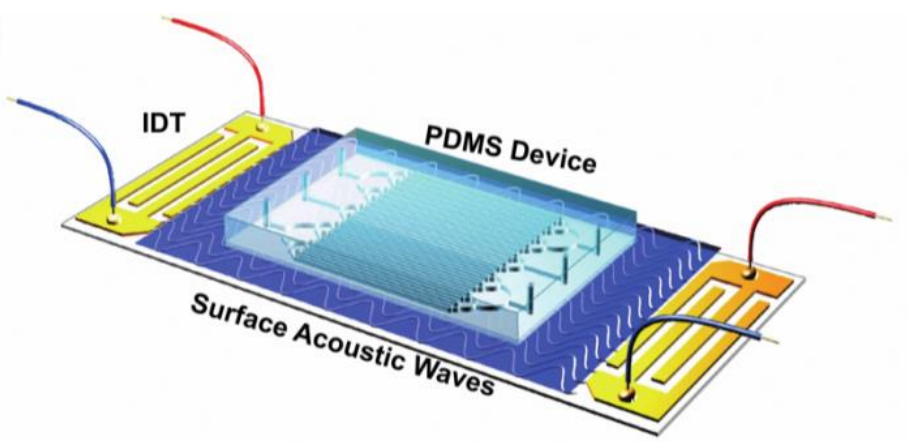

(c)

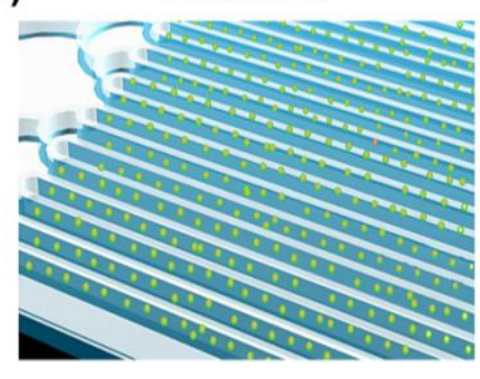

(b) Acoustic Cell Assembly

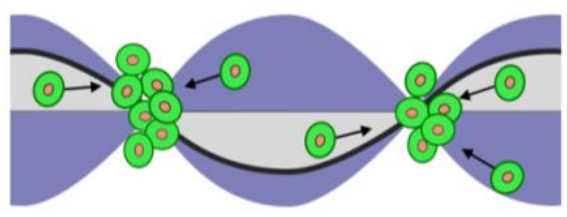

(e)

Culture

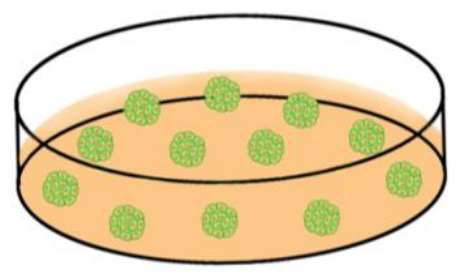

Fig.1. Working mechanism of acoustofluidic cell spheroid fabrication. (a). Schematic plot of highthroughput acoustofluidic cell spheroid assembly device. This device contains a disposable (polydimethylsiloxane, PDMS) device with multiple cell assemble channels and a reusable surface acoustic wave generator. (b) Schematics show acoustic cell assembly. The cells were randomly distributed in the microchannel without acoustics (c), aggregated into cell clusters with acoustics (d), and then transferred and cultured in an ultra-low attachment dish after an on-chip incubation (e). 
(a)

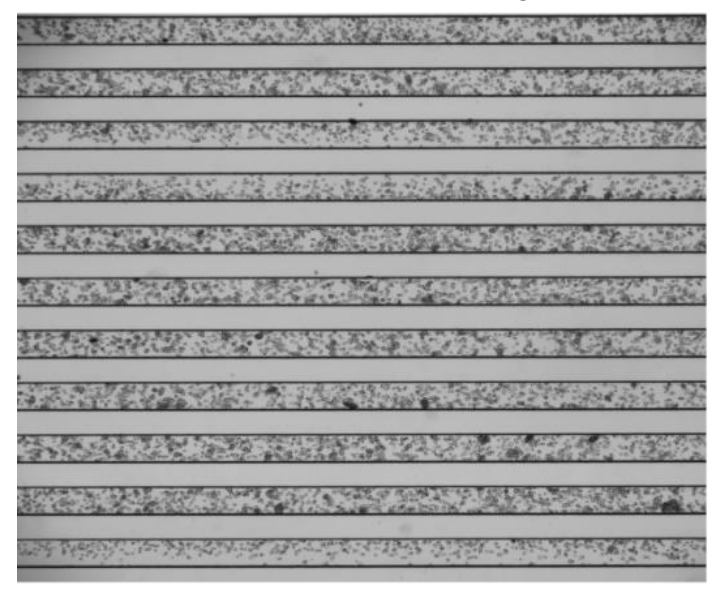

(c)

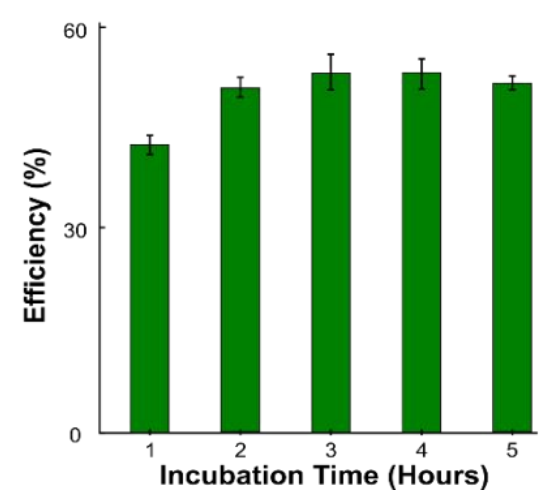

(b) After Acoustic Assembly

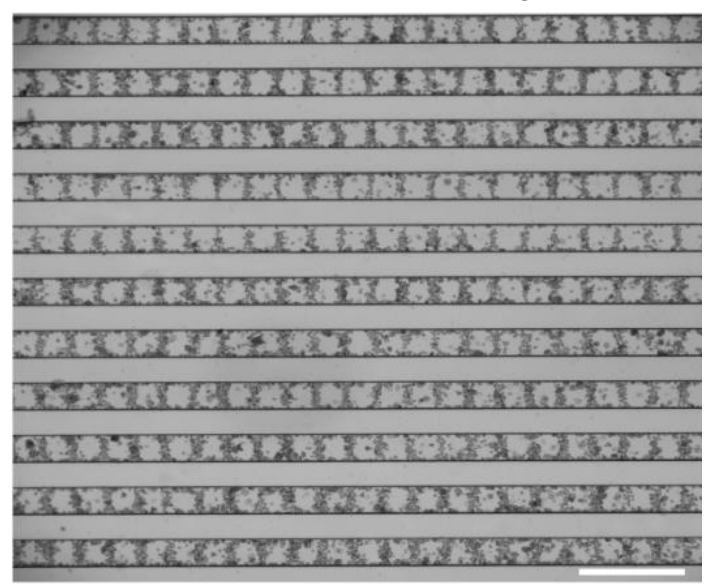

(d)

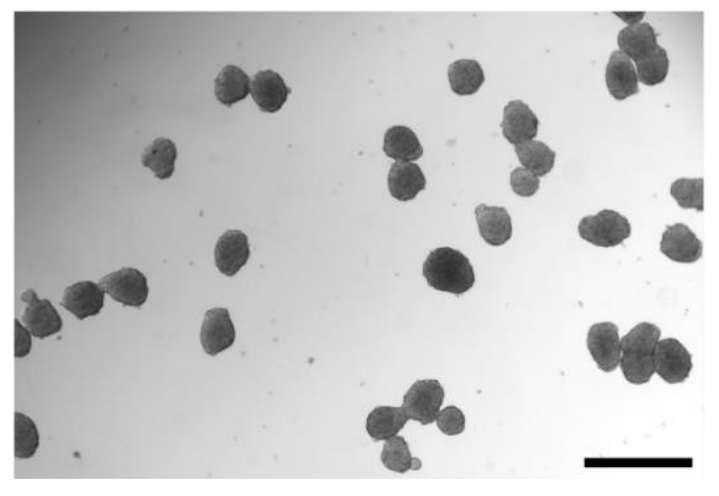

Fig.2. High throughput and short time cell spheroid formation. (a)-(b). Microscopic images of large-sale acoustofluidic assembled of cells before(a) and after(b) in the PDMS device. (c). The dependence of spheroids formation efficiency on on-chip incubation time. (d) Panc02 cell spheroid cultures in the ultra-low attachment petri-dish after one day culture. Scale bar: $500 \mu \mathrm{m}$ 


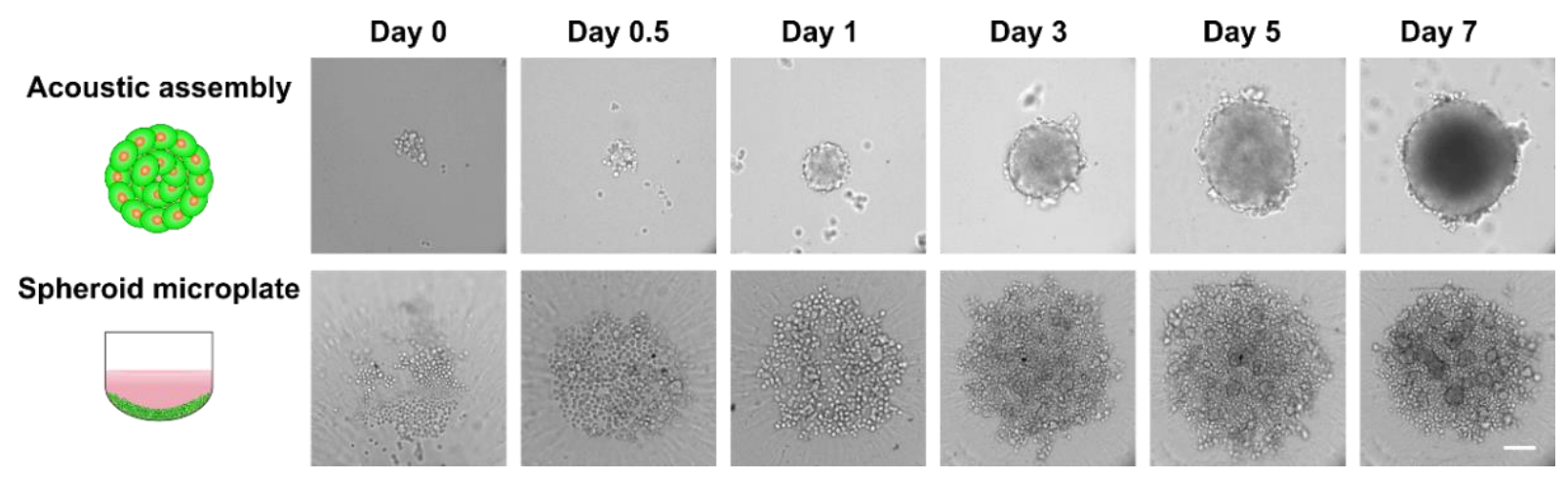

Fig.3. Acoustofluidic spheroid assembly vs Corning® spheroid microplate. Images of spheroid cultures at day 0 , day 0.5 , day 1 , day 3 , day 5 and day 7 were acquired using a microscope. Panc02 cell spheroids were formed around 0.5 day with our acoustic assembly method, while the Panc02 cell spheroids were not very well generated for 3 days with spheroid microplate method. Scale bar: $100 \mu \mathrm{m}$ 

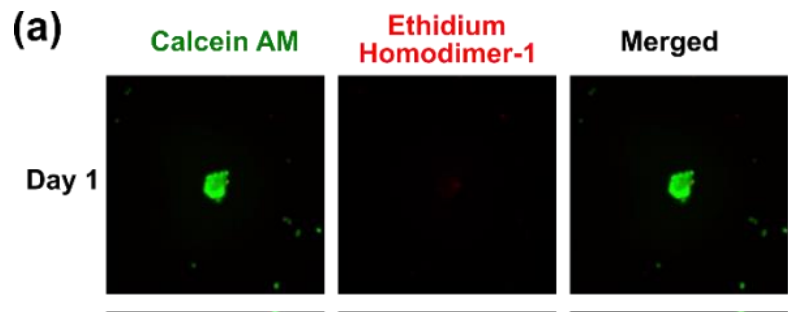

(b)
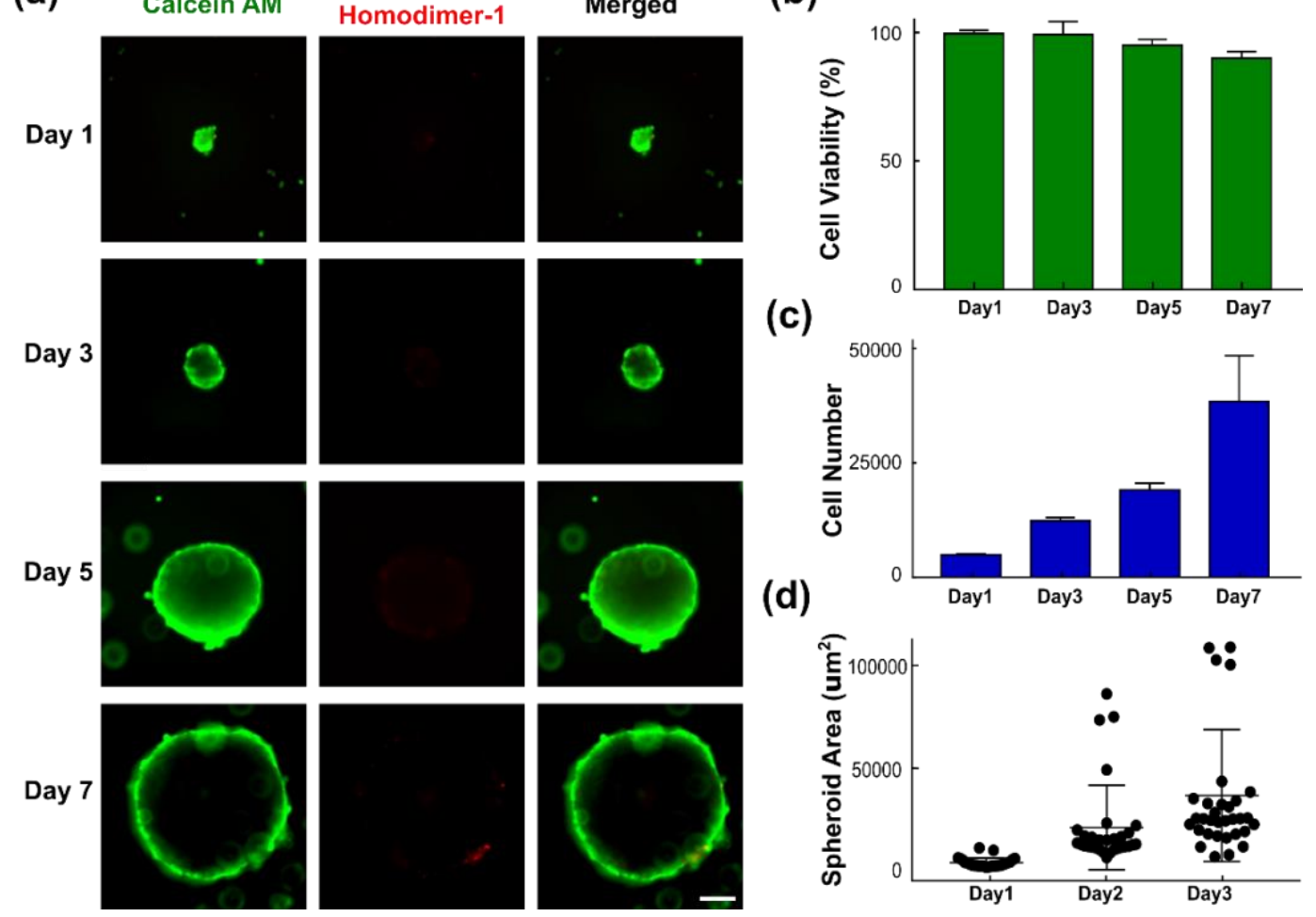

(c)

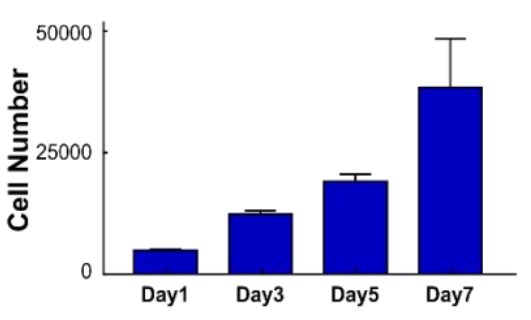

(d)

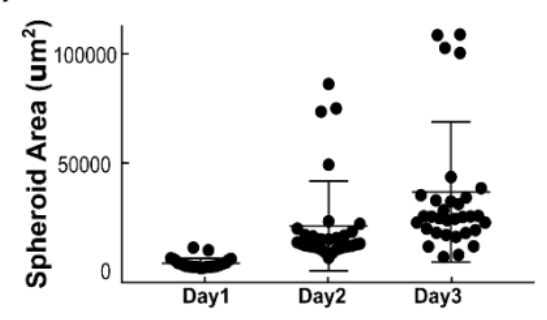

Fig.4. Cell spheroid characterization. (a). Panc02 cell spheroids were cultured in an ultra-low attachment dish for one week. Live cells were labeled with Calcein AM (green), and dead cells were labeled with ethidium homodimer-1(red). (b). The viability of Panc02 cells in the spheroids from day 1 to day 7. (c). Cell proliferation test of Panc02 cell spheroids with CCK-8 kit. (d). The sizes of Panc02 cell clusters and spheroids were measured from day 1 to day 3 . Scale bar: 100 $\mu \mathrm{m}$ 


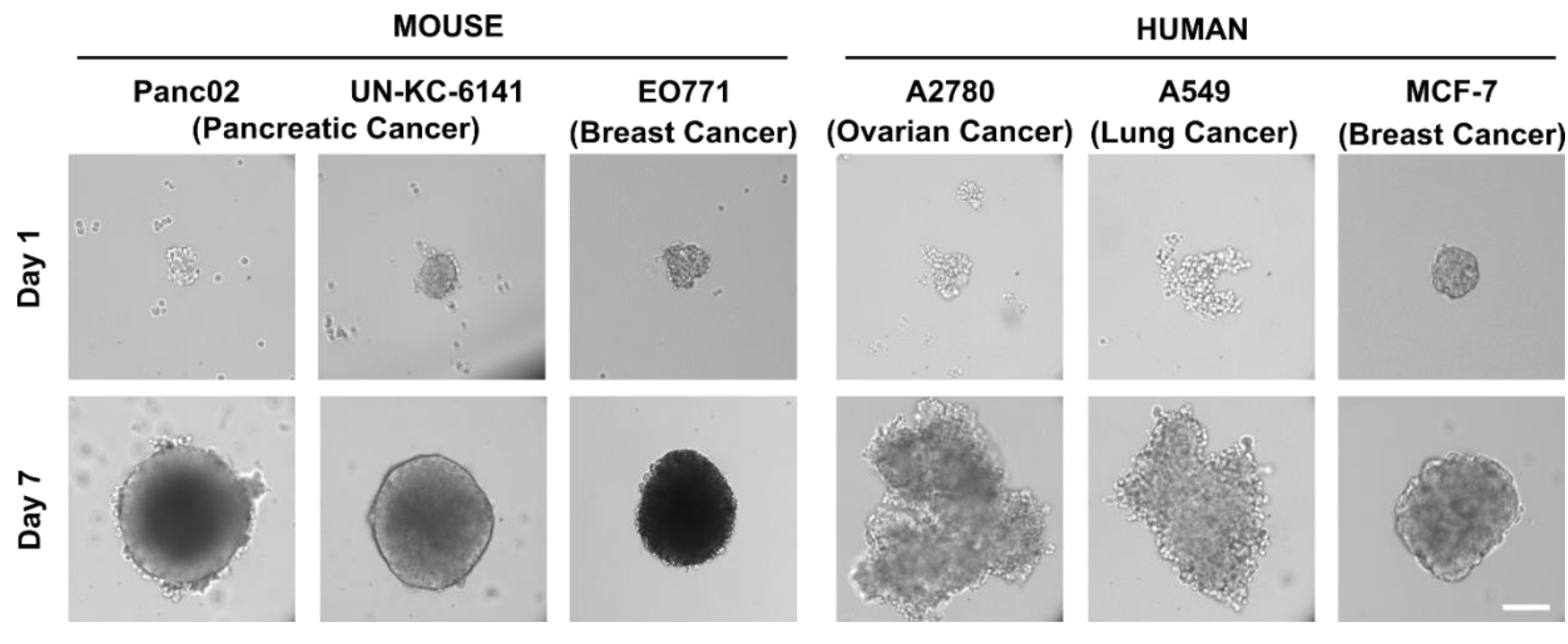

Fig.5. Cell spheroid formation with different cell types using our acoustofluidic method. Panc02, UN-KC-6141, EO771 and MCF-7 cell spheroids were round and compact after 7 days of culture. While A2780 andA549 formed only loose aggregates of cells after 7 days of culture. Scale bar: $100 \mu \mathrm{m}$ 

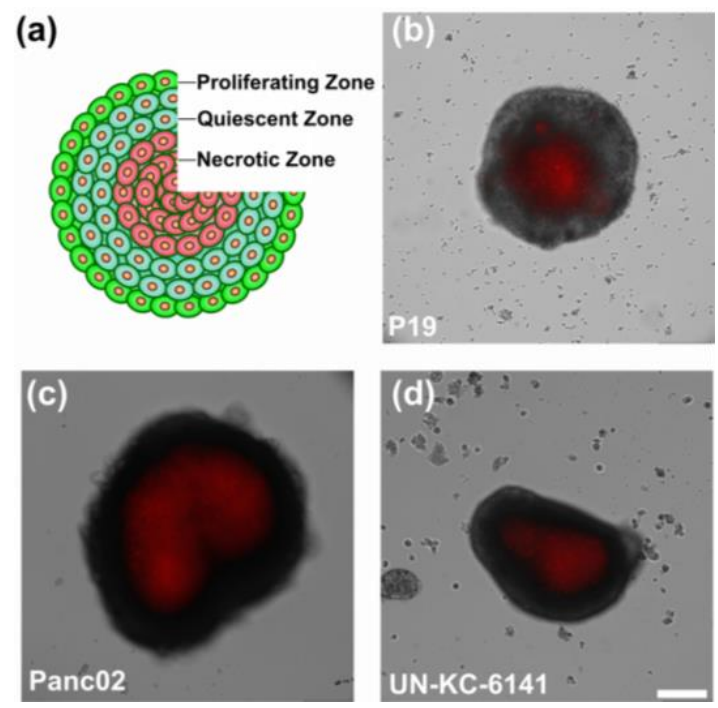

Fig.6. Hypoxic core of tumor spheroids. (a). Schematics shows the three-layered structure of the tumor spheroids including proliferating zone, quiescent zone, and necrotic zone (from outside to inside). (b)-(d) Hypoxic regions (in red) of P19 cell spheroid (b), Panc02 cell spheroid (c), and UNKC-6141 cell spheroids(d). Scale bar: $200 \mu \mathrm{m}$ 


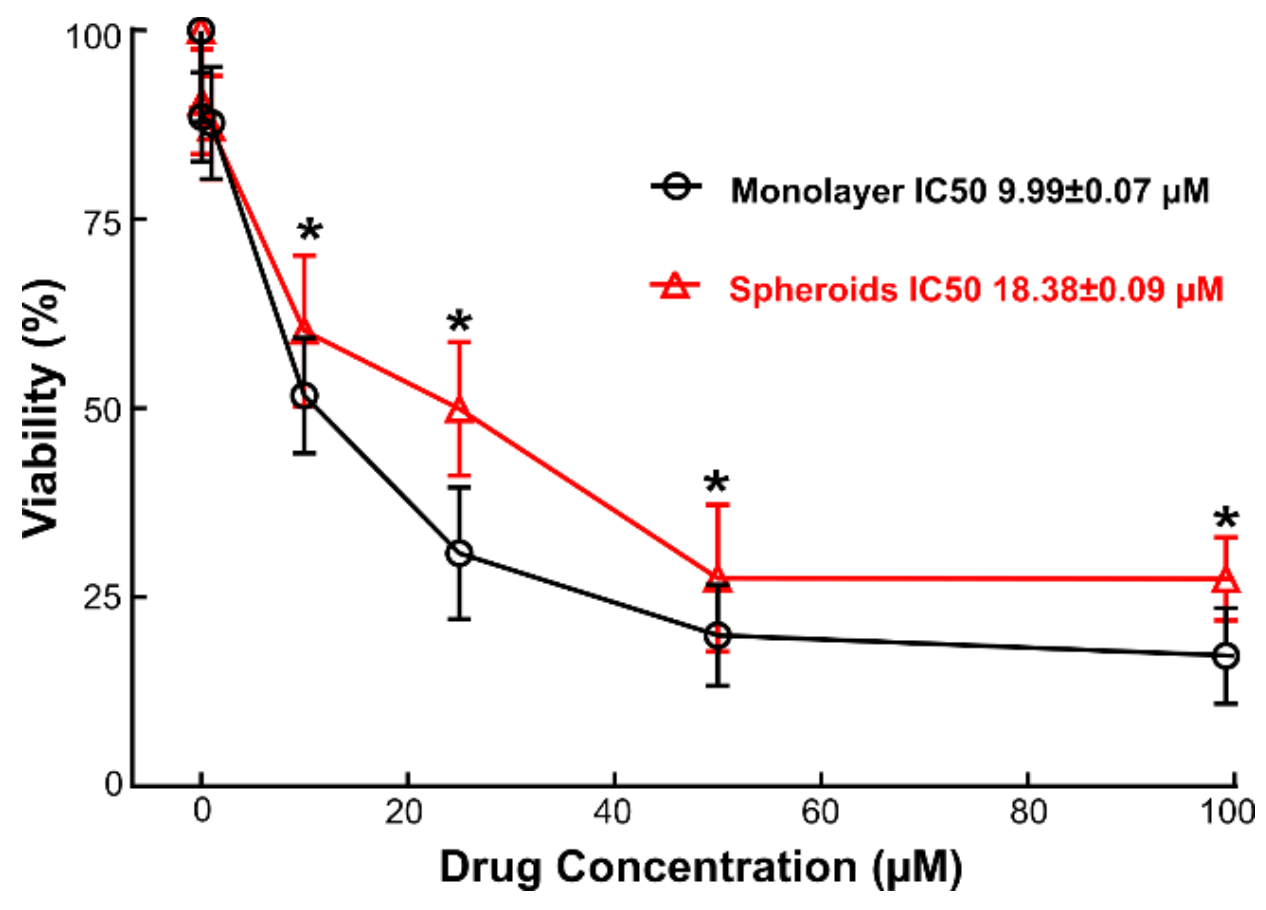

Fig.7. The drug (gemcitabine) resistance comparison between the monolayer cultures (black) and spheroid models (red) using MTT assays. Monolayer and spheroidal cultures of Panc02 cells were exposed to gemcitabine for 2 days. The results were presented as means \pm S.E.M of three independent experiments. $\left({ }^{*} P<0.001\right)$ 
Table 1. Cell spheroid formation methods

\begin{tabular}{|l|c|c|c|c|}
\hline \multicolumn{1}{|c|}{ Technique } & $\begin{array}{c}\text { Time required } \\
\text { [Day] }\end{array}$ & $\begin{array}{c}\text { Cell Number } \\
\text { required [×106] }\end{array}$ & $\begin{array}{c}\text { Number of } \\
\text { spheroids }\end{array}$ & $\begin{array}{c}\text { Uniformity } \\
\text { Control }\end{array}$ \\
\hline Acoustic assembly & 1 & 0.2 & High $(>6,000)$ & Yes \\
\hline Spheroid microplate63 & $3-7$ & 0.5 & Low (96-384) & No \\
\hline Hanging drop17 & 7 & 0.5 & Low (96-384) & Yes \\
\hline Magnetic levitation64 & 7 & 0.5 & Low (96) & Yes \\
\hline Spinner-flask65 & $14-35$ & 5 & High & No \\
\hline
\end{tabular}

